Rev. Colomb. Nefrol. 2017;4(2): 217 - 225 http://www.revistanefrologia.org

doi: http://dx.doi.org/10.22265/acnef.4.2.278

Reporte de caso

\title{
Riñón en herradura
}

\author{
Horseshoe Kidney
}

\section{Catalina Vásquez-Guarín', Frank Rojo-González², Juan Ríos-Salazar³,", Karen Ruiz-Naranjo³, Jineth Carolina Sarmiento-Rodríguez ${ }^{3}$}

\begin{abstract}
${ }^{1}$ Grupo Regional Noroccidente de Patología, Antropología e Identificación Forense, Instituto Nacional de Medicina Legal y Ciencias Forenses, Medellín, Colombia.
${ }^{2}$ Asistente Forense, Instituto Nacional de Medicina Legal, Medellín, Colombia.
\end{abstract}

${ }^{3}$ Estudiante, Programa de Medicina, Facultad de Ciencias de la Salud, Corporación Universitaria Remington, Medellín, Colombia.

\section{Resumen}

Se expone el caso de un hombre de 39 años que presenta muerte violenta en la ciudad de Medellín. Durante la necropsia se encuentra un riñón en herradura, que no tuvo diagnóstico clínico en su momento, según los hallazgos en su historial médico. Es un caso que puede tener implicaciones en el campo médico, dada la vasculatura accesoria que presenta, presentación que no es comúnmente observada. La falta de sintomatología y su hallazgo espontáneo durante la necropsia hacen ver que, a pesar de la poca incidencia, no es extraño encontrar el caso en pacientes de nuestro entorno. Este tiene mayor impacto en el campo quirúrgico y, en ocasiones, en las afecciones urinarias.

Metodologia: descriptivo. Se obtuvo consentimiento informado, de la familia del occiso, para el estudio de su historial médico.

Palabras clave: Variante anatómica, riñón en herradura, renal, riñón, circulación renal, función renal, anastomosis.

doi: http://dx.doi.org/10.22265/acnef.4.2.278

\section{Abstract}

We present the clinical case of a 39 year old male that presented with a violent death in the city of Medellin. During the necropsy, a horseshoe Kidney was found, which was never diagnosed according to the patients' medical history. It is a case relevant in the medical field given the unusual vasculature that was found, because it's not a usual arrangement. The lack of symptoms and its spontaneous finding during the necropsy, shows us that it's not a rare find in our environment and that it has a big impact in the surgical fields, and in occasions, with urinary tract infections.

Methodology: Descriptive. Informed consent was given by the deceased relatives for the use and analysis of the medical history.

Key words: Anatomic variant, horseshoe kidney, renal, kidney, renal blood flow, renal function, anastomosis.

doi: http://dx.doi.org/10.22265/acnef.4.2.278

\section{c) $(\$)$}

Referenciar este artículo: Vásquez-Guarín C, Rojo-González F, Ríos-Salazar J, Ruiz-Naranjo K, Sarmiento-Rodríguez JC. Riñón en herradura. Reporte de caso. Rev. Colomb. Nefrol. 2017;4(2): 217-225 doi: http://dx.doi.org/10.22265/acnef.4.2.278

Correspondencia: *Juan Ríos-Salazar, pit_84@live.com

Recibido: 20-05-17 - Aceptado: 17-07-17 • Publicado en línea: 27-07-17 


\section{Introducción}

$\mathrm{E}$ 1 riñón en herradura es una anomalía anatómica que se presenta aproximadamente en uno de cada 400-600 casos, principalmente en hombres. Es la anomalía de fusión más común, aunque es relativamente infrecuente. Las principales complicaciones están relacionadas con la severidad de la fusión renal, como hidronefrosis, infecciones, litiasis, dolor abdominal y, en algunos casos, masas abdominales. El mayor reto que se presenta con esta variación anatómica está en el campo quirúrgico, dadas sus múltiples presentaciones morfológicas y vasculares ${ }^{1,2}$.

\section{Descripción del caso clínico}

Se recibe para necropsia un cadáver de hombre no identificado, de 40 a 45 años de edad, quien posteriormente se identifica, de manera fehaciente, como DRP* de 39 años.

Al examen externo, se encuentra un hombre de apariencia cuidada, contextura mediana, adecuadamente vestido, la ropa presenta sangre en la camisa parte anterior y posterior. No se hallan señales particulares como cicatrices, lunares, ni tatuajes.

Se observan, como signos de violencia, un orificio de entrada por proyectil de arma de fuego en línea medio clavicular derecha a nivel del quinto espacio intercostal, con orifico de salida en región infraescapular del lado derecho; orificio de entrada en región temporal derecha, con orificio de salida en región parieto temporal del lado izquierdo.

Durante el examen interno del cuerpo y luego de haber realizado la evisceración del bloque tóraco abdomino pélvico, se presenta hacia su cara posterior y se da un hallazgo a nivel abdominal compatible con lo que se denomina riñón en herradura.

En una revisión macroscópica, sin realizar disección de los riñones, se observa que la unión de los mismos está dada en los polos superiores que se en- cuentran por delante de la arteria aorta abdominal. Al parecer, sin generar ninguna clase de alteración vascular a nivel del tronco celiaco.

Las medidas del espécimen anatómico descrito son las siguientes:

- Riñón derecho presenta una longitud de 15 $\mathrm{cm}$ hasta el polo superior, donde se encuentra la anastomosis de los dos riñones. El mismo presenta una circunferencia de $11 \mathrm{~cm}$ a nivel del íleo renal.

- Riñón izquierdo presenta una longitud de 16 $\mathrm{cm}$ hasta el polo superior, donde se encuentra la anastomosis de los dos riñones. Se encuentra una circunferencia de $10 \mathrm{~cm}$ a nivel del íleo renal.

Luego se verifica el trayecto de los uréteres, desde el íleo renal hasta la desembocadura en la vejiga, en el trayecto anteriormente descrito. No se encuentra doble sistema colector. Sin embargo, sí se describe que cada riñón cuenta con su uréter único saliendo desde su íleo.

A nivel vascular, se puede describir que las arterias renales estaban en su posición usual, es decir, cada una se origina desde la aorta abdominal. No obstante, llama la atención que el sitio donde se encuentran unidos los riñones (a nivel de los polos superiores) presenta una arteria accesoria, la cual se origina desde la aorta abdominal en su cara dorsal. Lo anterior nos indica que, en este caso en particular, los riñones cuentan, no con dos, sino con tres arterias para nutrir todo el componente vascular de esta variante anatómica.

En el sistema venoso se encuentra que, en los polos superiores renales (donde se describe la anastomosis), se presentan como variante anatómica dos venas acompañando la arteria accesoria. Dichas venas no desembocan en la vena cava, sino que se anastomosan, casi de forma inmediata, después de salir del íleo accesorio y desemboca a nivel de la vena renal del lado derecho. 
Las glándulas suprarrenales se encontraban en posición usual, descrita de la siguiente manera:

- La glándula suprarrenal derecha se encontraba adosada a la cara inferior del lóbulo derecho del hígado y en relación con el polo superior.

- La glándula suprarrenal izquierda se encontraba en relación con el polo superior en su tercio terminal y sobre el íleo renal del mismo lado. Es importante resaltar que, aunque la disposición anatómica es la usual, la glándula se encuentra mucho más cerca del íleo de lo habitual.

Se solicitó información del historial médico, previo consentimiento informado de los familiares. Después de revisar la historia clínica en su totalidad, no se encontró ninguna descripción, ni examen, que permitieran conocer con antelación que el occiso presentaba esta variante anatómica. Llama la atención que, en la revisión de la historia clínica, se encuentra que el paciente consultó, en varias oportunidades, por infecciones del tracto urinario a repetición, que se resolvían con tratamiento antibiótico usual. En los paraclínicos de función renal no se encuentra ninguna anormalidad y los niveles de creatinina se presentan en los niveles esperados para la edad; el citoquímico de orina no presenta ninguna anormalidad.

La persona en vida presentaba cifras de presión levemente elevadas, sin indicación para inicio de manejo farmacológico. Solo se prescribían cambios en el estilo de vida.

Después de concluir la necropsia, los hallazgos permitieron concluir que el deceso de quien en vida correspondía al nombre de DRP de 39 años, se debió a:

- Manera de muerte: heridas por proyectil de arma de fuego.

- Mecanismo de muerte: violenta - homicidio.

- Causa de muerte: laceración encefálica secundaria e heridas por proyectil de arma de fuego.

\section{Discusión}

\section{Anatomía normal del riñón}

Los riñones miden aproximadamente 10 centímetros de largo, 5 centímetros de ancho y 2,5 $\mathrm{cm}$ de grosor. Se sitúan retroperitonealmente en la pared posterior del abdomen, uno a cada lado de la columna vertebral, a nivel de las vértebras T12-L3. Superiormente, las caras posteriores de los riñones se relacionan con el diafragma, que los separa de las cavidades pleurales y del decimosegundo par de costillas ${ }^{3}$.

La cara superomedial de cada riñón está en contacto con una glándula suprarrenal, las cuales actúan como parte del sistema endocrino. Los órganos urinarios superiores (riñones y uréteres), sus vasos y las glándulas suprarrenales son estructuras retroperitoneales. En el borde medial cóncavo de cada riñón está el hilio renal, entrada al seno renal. Las estructuras como vasos, nervios y estructuras que drenan la orina de los riñones cruzan el seno renal a través del hilio renal. Posteriormente, las porciones superiores de los riñones se sitúan profundas respecto a las costillas 11 y $12^{3}$.

En el borde medial cóncavo de cada riñón está el hilio renal, como mencionamos, entrada al seno renal. Las estructuras como vasos, nervios y estructuras que drenan la orina de los riñones cruzan el seno renal a través del hilio renal. En el hilio, la vena renal es anterior a la arteria renal, que a su vez es anterior a la pelvis renal. Dentro del riñón el seno renal está ocupado por la pelvis, cálices, vasos y nervios renales. La pelvis renal es la expansión aplanada y en embudo del extremo superior del uréter. El vértice de la pelvis renal se continúa con el uréter. La pelvis renal recibe dos o tres cálices mayores, cada uno de los cuales se divide en dos o tres cálices menores. Cada cáliz menor está formado por una papila renal, el vértice de la pirámide renal desde donde se excreta la orina ${ }^{3}$.

\section{Uréteres}

Son conductos musculares cuya longitud es de 25 a $30 \mathrm{~cm}$, con una luz estrecha, que trasportan la 
orina de los riñones a la vejiga urinaria. Los uréteres discurren inferiormente desde los vértices de la pelvis renal en los hilios de los riñones, y pasan sobre la línea terminal al nivel de la bifurcación de las arterias iliacas comunes. Luego, discurren a lo largo de la pared lateral de la pelvis y entran en la vejiga urinaria. Las porciones abdominales de los uréteres se adhieren estrechamente al peritoneo parietal y son retroperitoneales a lo largo de su recorrido ${ }^{3}$.

\section{Glándulas suprarrenales}

Se localizan entre la cara superomedial de los riñones y el diafragma. Están rodeadas por la fascia renal, mediante la cual se unen a los pilares del diafragma. Están separadas de los riñones por un delgado tabique ${ }^{3,4}$.

La forma y las relaciones de las suprarrenales difieren en ambos lados. La glándula derecha, de forma piramidal, es más apical respecto al riñón izquierdo, se sitúa anterolateral al diafragma y está en contacto con la vena cava inferior antero medialmente y con el hígado antero lateralmente. La glándula izquierda, de forma semilunar, es medial a la mitad superior del riñón izquierdo y se relaciona con el bazo, el estómago, el páncreas y el pilar izquierdo del diafragma. Cada suprarrenal tiene un hilio por el cual abandonan la glándula las venas y vasos linfáticos, mientras que las arterias y los nervios entran en ella por numerosos puntos ${ }^{3,4}$.

Las glándulas suprarrenales tienen dos partes: la corteza y médula. La corteza secreta cortico esteroides y andrógenos. Estos permiten que el riñón retenga sodio y agua en respuesta al estrés, aumentando el volumen y la presión de la sangre. La medula suprarrenal es una masa de tejido nervioso invadida por capilares y sinusoides. Está formada por células cromafines que segregan catecolaminas, especialmente adrenalina, al torrente sanguíneo en respuesta a estímulos de las neuronas pre sinápticas ${ }^{3,4}$.

\section{Vasos y nervios de los riñones, uréteres, y glándulas suprarrenales}

La arteria renal derecha pasa posterior a la vena cava inferior. Cada arteria se divide cerca del hilio en cinco arterias segmentarias. Estas son arterias terminales, es decir, que no se anastomosan con otras arterias segmentarias. Las arterias segmentarias se distribuyen hacia los segmentos del riñón, así:

- El segmento superior (apical) está irrigado por la arteria segmentaria superior (apical); los segmentos anterosuperior y anteroinferior están irrigados por las arterias segmentarias anterosuperior y anteroinferior; el segmento inferior está irrigado por la arteria segmentaria inferior. Estas arterias se originan en la rama anterior de la arteria renal.

- La arteria segmentaria posterior se origina en una prolongación de la rama posterior de la arteria renal e irriga el segmento posterior del riñón ${ }^{3}$.

Las venas renales drenan los riñones y se unen de forma variable para formar las venas renales derecha e izquierda.

\section{Irrigación arterial y drenaje venoso de uréteres}

De las arterias renales nacen ramas arteriales para la porción abdominal del uréter y hay otras ramas menos constantes de la arteria testicular u ovárica, la aorta abdominal y las arterias iliacas comunes. Las venas que drenan la porción abdominal de los uréteres drenan en las venas renales y gonadales ${ }^{3}$.

\section{Arterias y venas suprarrenales}

Las arterias suprarrenales proceden de tres fuentes:

- Arterias suprarrenales superiores proceden de las arterias frénicas inferiores.

- Arterias suprarrenales medias, de la aorta abdominal.

- Arterias suprarrenales inferiores, de las arterias renales.

El drenaje venoso se realiza en la vena suprarrenal ${ }^{3,4}$. 


\section{Nervios de los riñones, los uréteres y las glándulas suprarrenales}

Los nervios de los riñones proceden del plexo nervioso renal y están formados por fibras simpáticas y parasimpáticas. El plexo nervioso renal recibe fibras de los nervios esplacnicos abdominopélvicos. Los nervios de la porción abdominal de los uréteres proceden de los plexos renal, aórtico abdominal e hipogástrico superior.

La rica inervación de las glándulas suprarrenales procede del plexo celiaco y de los nervios esplacnicos abdominopelvicos ${ }^{3}$.

\section{Riñón en herradura}

El riñón presenta diferentes variaciones anatómicas, según describen Watson y Harper, tales como riñón pélvico, riñón cruzado ectópico, agenesia renal, riñón en herradura. También puede presentar variaciones vasculares como múltiples arterias, sobreposición de la arteria renal derecha pasando sobre la vena cava, vena renal izquierda retro-aortica, entre otras ${ }^{4}$.

El riñón en herradura es una de las anomalías anatómicas más comunes y se refiere a la fusión lateral de éstos. Es más frecuente en los hombres y el istmo puede estar formado por una banda fibrosa o parénquima funcional ${ }^{5,6,7}$.

Existen varias formas de clasificar al riñón en herradura: por su presentación macroscópica y lugar de fusión; por su clínica; o por su distribución vascular.

Según el lugar en el que se presente la fusión:

- Riñón en herradura "en U”: presenta fusión medial y se ubica de forma simétrica a ambos lados de la columna vertebral.

- Riñón en Herradura "en L": resulta de la fusión lateral entre un riñón horizontal y otro vertical, y tiene el istmo lateral a la línea medias.

El riñón en herradura puede estar a cualquier altura del trayecto normal que se espera del ascenso de los riñones, pero se encuentran principalmente en la porción baja de la columna lumbar. Esto se debe a la restricción del ascenso a la altura del origen de la arteria mesentérica inferior, aproximadamente entre L3 y L5, y anterior a la aorta abdominal y la vena cava inferior. Los uréteres generalmente pasan por la sección anterior de los polos fusionados y bajan de forma medial. Pueden tener irrigación arterial proveniente de las arterias iliacas, sacras, mesentéricas o aorta ${ }^{5,6,7}$. (figura 1)

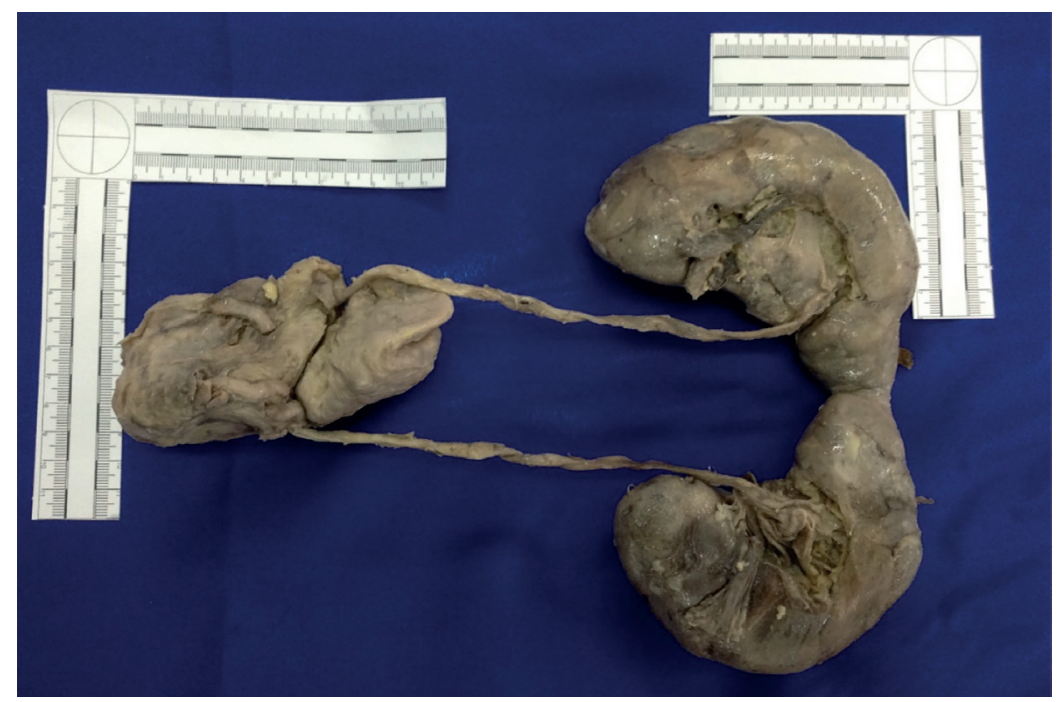

Figura 1. Vista espécimen completo de riñón en herradura con vías urinarias, vejiga y próstata. 
Clínicamente, lo podemos clasificar en 3 grupos:

I. Anomalía presente, pero sin cambios patológicos y sin sintomatología. No hay hidronefrosis ni complicaciones ya que la unión ureteropliélica es amplia.

II. Anomalía presente sin alteración patológica, pero el paciente se queja de síntomas como dolor abdominal. Causado por compresión de la aorta abdominal. Hay compresión vascular y nerviosa y múltiples alteraciones urinarias como litiasis o infecciones frecuentes. Esta anomalía se conoce como el síndrome de Rovsing o Enfermedad del riñón en herradura. El dolor se exacerba cuando se sienta después de estar en posición supina, o cuando pasa de estar sentado a ponerse de pie.

III. Se presenta la anomalía con complicaciones. Los síntomas son por estados patológicos. Debido a la ubicación posterior de los cálices hay estrechez ureteropiélica. No hay síntomas individuales o en grupo que indiquen cambios específicos que nos distingan entre un riñón en herradura o uno normal. Solo se puede determinar esta anomalía por métodos urológicos de diagnóstico como la urografía excretora y la pielografía retrograda, TAC, sonografia y angiografía.

Para una mejor organización, se han establecido 6 patrones básicos que permiten clasificar los diferentes tipos de irrigación:

- 1a: todos los segmentos del riñón en herradura están irrigados por una arteria única, exceptuando la circulación colateral, que puede tener una sola arteria renal para esta función.

- 1b: los segmentos superior y medio pueden tener una arteria renal única, con una rama de la aorta abdominal para la irrigación arterial de los segmentos inferiores.

- 1c: los segmentos inferiores son irrigados por arterias que surgen de un tronco común, proveniente de la aorta abdominal, y los troncos superior y medio son irrigados por una arteria renal para cada lado.

- 1d: los segmentos superiores y medios tienen irrigación de múltiples arterias renales.

- 1e: el istmo también tiene irrigación de arterias que salen por debajo del mismo, provenientes por lo general de la aorta abdominal, ya sea independientes, o de un tronco común.

- 1f: los segmentos inferiores fusionados pueden tener irrigación de las ramas de la arteria iliaca común, o en casos menos comunes, de la arteria iliaca interna o arteria sacra media ${ }^{7,8}$. (figura 2)

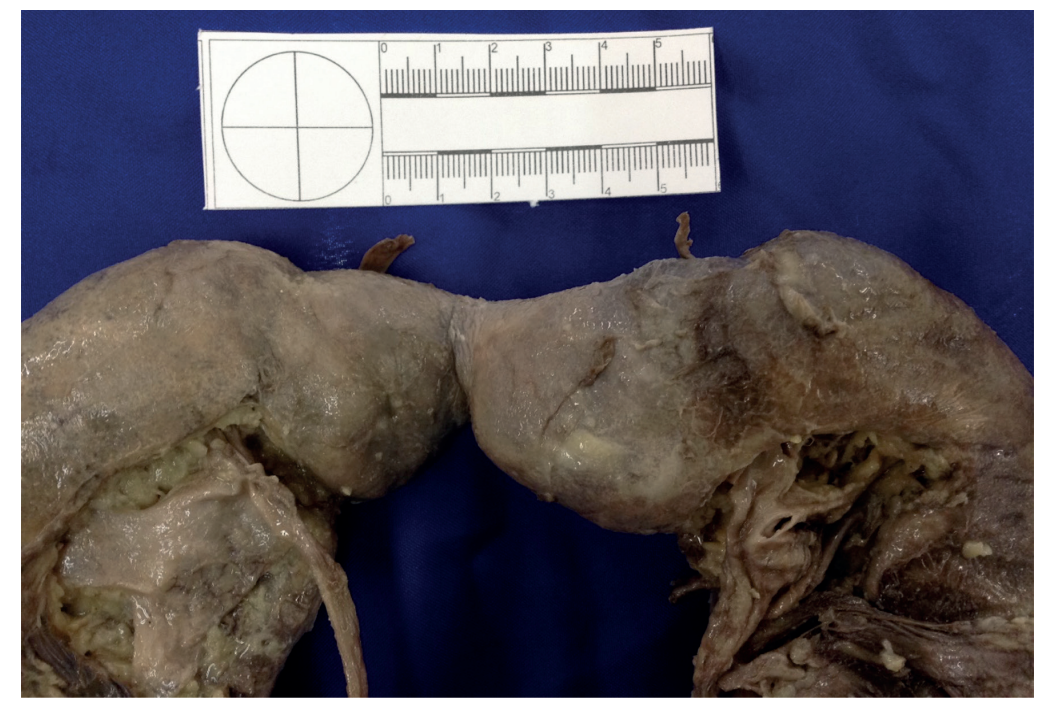

Figura 2. Detalle anatómico del hilio renal y arteria accesorias en polos inferiores de los riñones. 
Se ha reportado que el riñón en herradura se asocia con anomalías caliciales, ureterales y vasculares. Puede tener irrigación normal de arterias renales accesorias, que se originan en diferentes niveles y entran por el hilio renal, y también arterias aberrantes, que entran directamente a los polos o el istmo del riñón. Con excepción de algunas de las arterias que entran al istmo, ningún vaso ha sido visto entrar al riñón por su cara dorsal.

El riñón en herradura está frecuentemente acompañado por anomalías de la vena cava inferior, (como la doble, izquierda, o pre-ístmica vena cava inferior), y su coexistencia probablemente se deba a su origen embrionario.

La posición anormal de los riñones junto a su forma los hace más susceptibles a daño severo después de algún tipo de trauma abdominal, ya que no quedan protegidas por las costillas ${ }^{8}$.

El riñón en herradura presenta síntomas generalmente atribuidos a las anomalías asociadas, como la hidronefrosis, infecciones y litiasis, aunque casi siempre es asintomático. El síntoma más observado es el dolor abdominal, que irradia hacia la columna lumbar. También se pueden presentar infecciones urinarias causadas por estasis urinaria. Se han referido síntomas gastrointestinales como dolor, náuseas y vómito. En ocasiones, se puede tener una masa a nivel abdominal, la cual se confirma con un pielograma intravenoso o una ecografía ${ }^{5,6,7,8}$. (figura 3)

\section{Conclusiones}

- Los hallazgos permiten determinar que la presencia de la variante anatómica del riñón en herradura no es tan inusual en nuestro medio.

- La mayoría de personas que presentan el riñón en herradura son asintomáticas la mayor parte de su vida.

- Las pruebas de función renal, en este caso específico, no muestran alteraciones fuera de lo usual para la edad.

- Las mayores complicaciones del riñón en herradura radican en el abordaje quirúrgico, cuando se encuentra como un hallazgo incidental, así como en en las pruebas de función excretora y su interpretación con esta variante.

- No existe claridad si, en este caso, el riñón en herradura está relacionado con la elevación de los niveles de presión arterial.

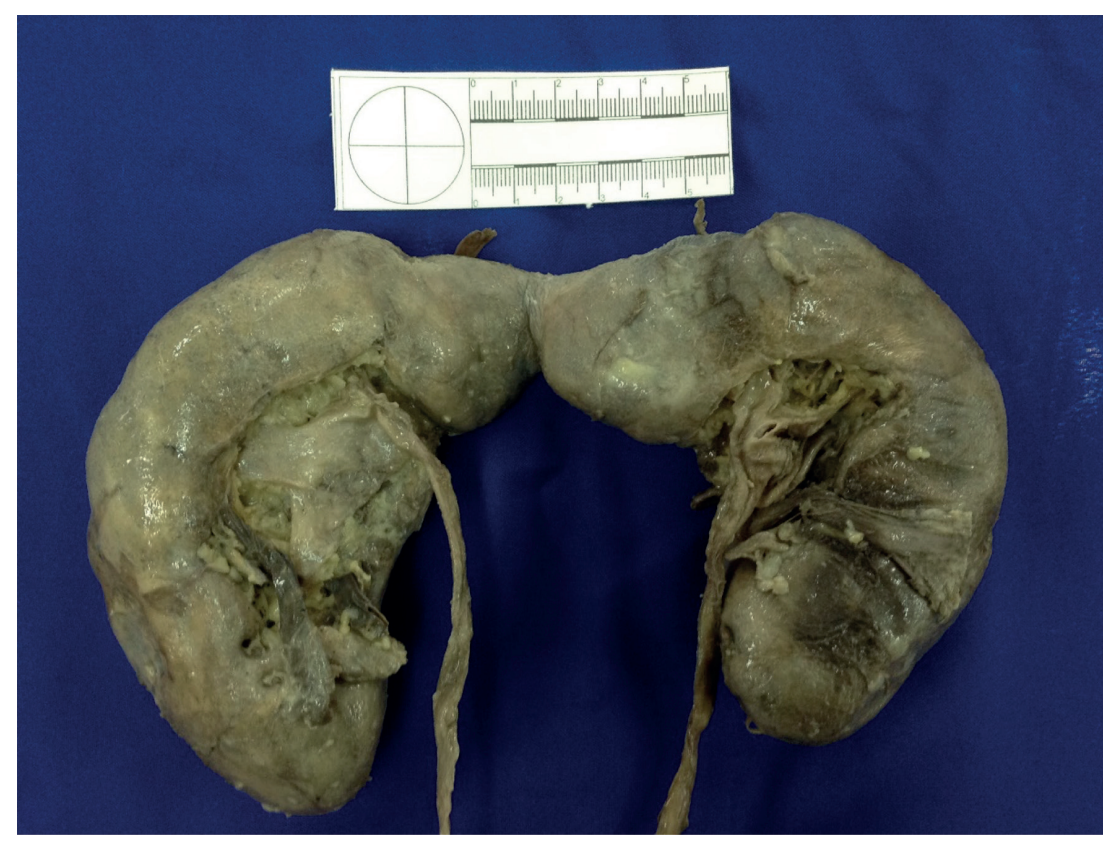

Figura 3. Detalle anatómico del hilio renal y arteria accesorias en polos inferiores de los riñones. 


\section{Conflicto de intereses}

Los autores declaran no tener conflicto de intereses en la realización de este artículo.

\section{Financiación}

El trabajo no recibió ningún tipo de financiación

\section{Contribución de los autores}

Catalina Vásquez: realizó el protocolo de necropsia.

Frank David Rojo: realizó la disección del espécimen.

Juan David Ríos, Karen Ruiz Naranjo y Carolina Sarmiento: realizaron la revisión bibliográfica. Entre todos los integrantes del grupo se realizó la redacción del texto.

\section{Responsabilidades éticas}

Protección de personas y animales

Los autores declaran que para esta investigación no se han realizado experimentos en seres humanos ni en animales.

\section{Confidencialidad de los datos}

Los autores declaran que en este artículo no aparecen datos de pacientes.

\section{Derecho a la privacidad y consentimien- to informado \\ Los autores declaran que en este artículo no aparecen datos de pacientes.}




\section{Referencias}

1- Watson C.J.E, Harper S.J.F. Anatomical Variation and Its Management in Transplantation. American Journal of Transplantation. (2015);15:1459-71. Disponible en: https://doi.org/10.1111/ajt.13310

2- Moore KL, Dalley AF, Agur AM. Abdomen. En: Gotzens García V. Anatomía con orientación clínica. 6ta ed. Philadelphia: Lippincott Williams \& Wilkins; 2010. p. 292-7.

3 George AL, Neilson EG. Biología celular y molecular de los riñones. En: Longo D. L. Harrison Principios de Medicina Interna. $18^{\mathrm{a}}$ edición. México: McGraw-Hill interamericana; 2012. p. 2280-8.

4 Natsis K., Piagkou M., Skotsimara A., Protogerou V., Tsitouridis I., Skandalakis P. Horseshoe Kidney: A review of anatomy and pathology. Surg Radiol Anat. (2014)Aug;36(6):517-26. Disponible en: https://doi.org/10.1007/s00276-013-1229-7

5 Sadler TW. Embriología médica. 11a ed. Philadelphia: Lippincott Williams \& Wilkins; 2010. Chapter 16, Sistema urogenital; p. 235-44.

6 Bouali O., Labarre D., Molinier F., Lopez R., Benouaich V., Lauwers F., Moscovici J. Anatomic variations of the renal vessels: focus on the precaval right renal artery. Surg Radiol Anat. (2012);34:441-6. Disponible en: https://doi.org/10.1007/s00276-011$\underline{0923-6}$

7 Pei Y., Shen H., Li J., Zhang H., Xia L., Wang L., Hu, D. Evaluation of renal artery in hypertensive patients by unenhanced MR angiography using spatial labeling with multiple inversion pulses sequence and by CT angiography. Am j Roentgenol (2012);199(5): 1142-8. Disponible en https://doi.org/ 10.2214/AJR.11.7263

8 Sakala M.D, Dyer R.B. The Horseshoe Kidney. Abdom Imaging. (2015);40(7):2910-1. Disponible en: https://doi.org/10.1007/ $\underline{\mathrm{s} 00261-015-0454-4}$ 